\title{
Effect of an $\alpha$-Tocopherol-Containing Antioxidant Parenteral Emulsion upon Gut Microbiota in Preterm Infants
}

\author{
Silvia Arboleya ${ }^{1}$, Clara G. de los Reyes-Gavilán ${ }^{1}$, Dimitris Konstantinou², \\ Maria Skouroliakou ${ }^{2,3}$ and Miguel Gueimonde ${ }^{1, *}$
}

${ }^{1}$ Probiotics and Prebiotics Group, Department of Microbiology and Biochemistry, Instituto de Productos Lácteos de Asturias (IPLA-CSIC), Villaviciosa, Asturias, Spain

${ }^{2}$ Department of Neonatology, IASO Maternity Hospital, Athens, Greece

${ }^{3}$ Department of Nutrition and Dietetics, Harokopio University, Athens, Greece

\begin{abstract}
Preterm babies are born with an immature intestine and are at higher risk for intestinal failure and needing for parenteral nutrition than full-term newborns. These babies show an altered intestinal microbiota, which may results of key importance for later health. However, there is a lack of information on effect of parenteral formulas upon the establishing infant microbiota. Moreover, the microbiota alterations present in preterm newborns have been related with an altered redox-potential which hinders the establishment of strict anaerobes. Thus, interventions aimed at reducing the oxidative stress in these infants, including those under parenteral nutrition, could also affect the intestinal microbiota composition. We assessed the effect upon the gut microbiota of the administration during 30 days of a lipid emulsion, containing $\omega 3$ polyunsaturated fatty acids and $\alpha$-tocopherol, or a control emulsion containing soybean-bases lipids to parenterally-fed preterm newborns. In spite of its high antioxidant potential, the infants receiving the experimental fat emulsion showed a trend to increase of facultative anaerobes such as enterobacteria and specifically of Klebsiella. This study stresses the need of further work monitoring the impact of early-life parenteral nutrition on the establishment of the intestinal microbiota.
\end{abstract}

Keywords: Preterm infants, Parenteral nutrition, lipid emulsion, Antioxidants, Intestinal microbiota.

\section{INTRODUCTION}

The basis for a healthy intestinal microbiota lies in the early neonatal period with the initial steps of the microbiota establishment [1]. In healthy infants the process starts with facultative anaerobes which reduce oxygen level allowing the establishment of strict anaerobic microbial populations [2]. During this relatively unstable and sensitive initial period any aberrancy in the microbiota development process may entail a high-risk of disease for later life [1,3]. Different factors influence the early establishment of the gut microbiota, and its further development, including gestational age, mode of delivery, antibiotic use or feeding habits, among others [3-5].

At birth the neonate is changed from a hypoxic fetal environment to a hyperoxic environment which causes oxidative stress and concomitant damage by reactive oxygen species. Preterm infants are born with an immature intestinal mucosa, which represents a risk for intestinal failure, and have been reported to have problems for properly managingoxidative stress due to their immature antioxidant systems [6]. This limited ability to manage the oxidative stress has been linked to the increased risk of the premature baby to suffer the

*Address correspondence to this author at the Department of Microbiology and Biochemistry of Dairy Products, Instituto de Productos Lácteos de Asturias (CSIC), Paseo Río Linares s/n, 33300 Villaviciosa, Asturias, Spain; Tel: +34 985892131; Fax: +34 985892233; E-mail: mgueimonde@ipla.csic.es so called "free radical-related diseases", such as retinopathy, bronchopulmonary dysplasia and necrotizing enterocolitis. For these reasons the supplementation of preterm babies diet with antioxidants has attracted the attention of researchers [7].

Recent data obtained from microbial cultureindependent techniques have identified clear alterations in the process of intestinal microbiota establishment in preterms when compared with that of full-term babies. In general preterm infants harbor higher levels of facultative anaerobes and reduced levels of strict anaerobes [4]. These observations suggest a deficiency in the development of a low redox-potential in the intestinal environment, which limits the establishment of the anaerobic gut microbiota in these babies. This has led to the hypothesis that the different microbiotas observed between preterm and full-term babies may be, at least partly, due to an altered intestinal redox environment [8]. Indeed, the redox potential has been reported as one of the major intestinal factors driving the microbiota composition [9]. Therefore, preterm infants would benefit from the development of strategies favoring the establishment of a healthy microbiota.

Moreover, in some cases, due to intestinal failure, the enteral feeding of the infant is not possible and parenteral nutrition $(P N)$ is needed. In spite of its 
important role for later health, little is known about the impact of PN upon the establishing gut microbiota. Animal studies in a PN associated liver injury model reported changes in the microbiota composition under different PN solutions [10]. However, the information on the impact of PN formulas on the preterm newborn microbiota is largely lacking.

This study aims at exploring the effect of PN upon the establishing gut microbiota in a group of preterm infants and to evaluate the impact of an antioxidant PN formula upon this process.

\section{SUBJECTS AND METHODS}

Twenty-five infants born at the "IASO" Maternity Hospital in Athens with gestational age $<32$ weeks, birth weight $<1500 \mathrm{~g}$, at least $80 \%$ of total energy intake as PN and admitted to the Neonatal IntensiveCare Unit (NICU) were included in the study. Infants with liver or bile duct disease were excluded from the study. The subjects participating in this work are a subset of a larger ongoing intervention study. The study was approved by the Scientific and Ethical Committee of the IASO Maternity Hospital.

At admission infants were randomly assigned to groups receiving either an $\omega 3$ polyunsaturated fatty acids containing formula supplemented with tocopherol (SMOFLipid 20\%, Fresenius Kabi HELLAS, Athens, Greece; medium-chain triglycerides [30\%], lipids from soybean oil ([0\%], olive oil [25\%], fish oil [15\%] $\alpha$ tocopherol $[0.2 \mathrm{mg} / \mathrm{mL}]$ ) (intervention group, $\mathrm{n}=8$ ) or a control emulsion containing soybean-based lipids (INTRALipid 20\%, Fresenius Kabi HELLAS, Athens, Greece) (control group, $n=17$ ) [7]. Infants started the PN from the first day of life, lipid emulsions (LE) were added in the PN solution from the second day of life. For all infants, PN regimens were designed and automatically produced as described previously [11]. Macronutrients were provided using the same products for glucose and amino acid solutions in both groups.

Fresh fecal samples were collected and immediately frozen at $-20^{\circ} \mathrm{C}$ until analyses. We monitored the fecal levels of seven intestinal microbial groups (Bacteroidaceae, Bifidobacteriaceae, Enterobacteriaceae, Enterococcaceae, Lactobacillus group and the genera Klebsiella and Weissella), as well as fecal short-chain fatty acids (SCFA) profile, during 30 days of administration to preterm infants of a parenteral LE. Bacterial levels of the different microbial groups were analyzed by quantitative PCR and SCFA concentrations were determined in fecal samples as previously described [4].

\section{RESULTS}

The results obtained showed a high inter-individual variability in both bacterial levels (Figure 1) and SCFA concentration (Figure 2) and were in the range of those normally observed for preterm infants $[4,5]$ with the

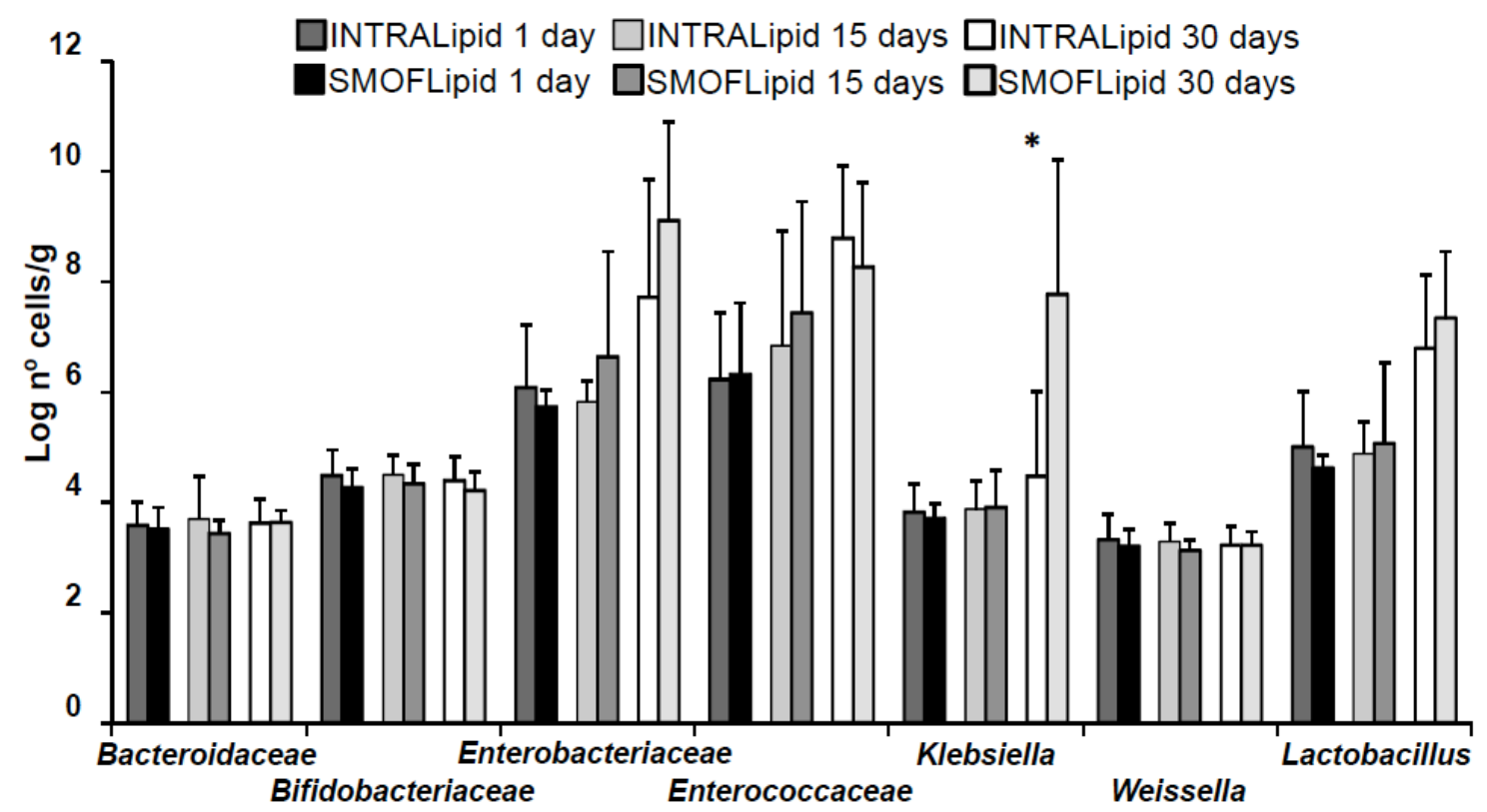

Figure 1: Levels of the different microbial groups analized by qPCR in feces of infants from both experimental groups (SMOFLipid and INTRALipid) at 1, 15 and 30 days of life.* Denotes statistically significant $(p<0.05)$ differences between both infant groups. 
sole exception of the unusually high levels of Klebsiella observed in the SMOF Lipid group at 30 days of age (Figure 1). In both experimental groups the levels of most facultative anaerobes (Enterobacteriaceae, Enterococcaceae or lactobacilli) increased along the study period whereas those of strictly anaerobic microorganisms (Bacteroides and Bifidobacterium) remained with only minor modifications. In spite of the high inter-individual differences, when the results from both infant groups were compared (U Mann-Whitney test) no differences were found at the beginning $\left(1^{\text {st }}\right.$ day of life) or after 15 days of intervention, but significantly higher $(p<0.05)$ levels of Klebsiella were found in the SMOF Lipid group at the end of the intervention (30 days). This observation was in good agreement with a trend (not significant) towards higher levels of Enterobacteriaceae in the SMOF Lipid group.

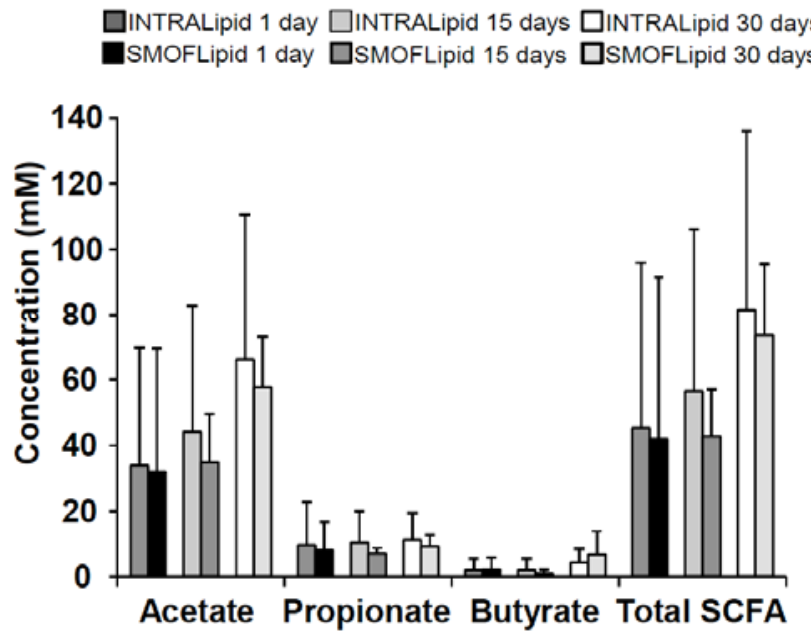

Figure 2: Concentration ( $\mathrm{mM}$ ) of the different short chain fatty acids (acetate, propionate and butyrate) as well as the sum of the three major SCFA, in feces of infants from both groups (SMOFLipid and INTRALipid) at 1, 15 and 30 days of life.

With regard to SCFA (Figure 2) the concentration of acetate and total SCFA increased along time whilst that of propionate and butyrate did not show major changes. When both infants groups were compared no statistically significant differences ( $p>0.05)$ were found between the intervention and control groups.

\section{DISCUSSION}

The SMOF Lipid emulsion used in this study has been previously found to improve the lipid profile, to reduce oxidative stress and to decrease bronchopulmonary dysplasia in preterm infants under PN [7, 12]. Reducing the levels of oxidative stress in these preterm infants may result beneficial for reducing the risk of disease [13]. Moreover, decreasing the intestinal oxidative stress could also influence the gut microbiota composition [8]. However, almost no information is available to date on the impact of $P N$ upon the gut microbiota establishment process and whether or not there is an effect of formulations with antioxidant properties.

We found an increase over time in the levels of facultative anaerobes, such as Enterobacteriaceae, Enterococacceae or the group of Lactobacillus, in both groups of infants during the first month of life. This finding illustrates that facultative anaerobes trend to increase in the microbiota of the parenterally-fed preterm babies, whilst the levels of the strict anaerobes analyzed, Bacteroides and Bifidobacterium, remained stable. Moreover, our results show an effect of the experimental fat emulsion on the establishing gut microbiota. In spite of being a formula with a high potential for reducing the oxidative stress, the levels of strict anaerobes remained unchanged whilst those of a facultative anaerobes such as enterobacteria and specifically of microorganisms of the genus Klebsiella increased along treatment when compared with the control emulsion. Oxidative stress is known to produce reactive oxygen species that may react with sulphides and amines present in the gut lumen to form S-oxydes and $\mathrm{N}$-oxydes, which in turn may be used as electron acceptors for anaerobic respiration by facultative anaerobes [14]. This may allow Enterobacteriaceae to out compete intestinal strict anaerobes, thus providing a reason for the increased levels of enterobacteria in the presence of oxidative stress in the gut [14]. However, in our study the oxidative status would be expected to be lower in the intervention group and, therefore, there is not a clear explanation for the increased levels of Klebsiella.

To date, very few information is available regarding the effect of PN and antioxidants upon the neonatal microbiota. The potentially different effects of antioxidants, together with the complexity of the fat emulsion used containing also $\omega 3$ fatty acids with antiinflammatory potential, and the lack of knowledge on the effect of $\alpha$-tocopherol on the microbiota hinders the interpretation of our results. Nevertheless, our work stresses the need for studies monitoring the impact of early-life PN on the establishing intestinal microbiota.

\section{ACKNOWLEDGEMENTS}

This work was funded by the Luis Noé Fernández Award from Fundación Alimerka (Spain). 


\section{REFERENCES}

[1] Conroy ME, Shi HN, Walker WA. The long-term health effects of neonatal microbial flora. Curr Opin Allergy Clin Immunol 2009; 9: 197-201.

http://dx.doi.org/10.1097/ACl.0b013e32832b3f1d

[2] Bezirtzoglou E. The intestinal microflora during the first weeks of life. Anaerobe 1997; 3: 173-7. http://dx.doi.org/10.1006/anae.1997.0102

[3] Penders J, Thijs C, Vink C, et al. Factors influencing the composition of the intestinal microbiota in early infancy. Pediatrics 2006; 118: 511-21. http://dx.doi.org/10.1542/peds.2005-2824

[4] Arboleya S, Binetti A, Salazar N, et al. Establishment and development of intestinal microbiota in preterm neonates. FEMS Microbiol Ecol 2012; 79: 763-72. http://dx.doi.org/10.1111/j.1574-6941.2011.01261.x

[5] Arboleya S, Sánchez B, Milani C, et al. Intestinal microbiota development in preterm neonates and effect of perinatal antibiotics. J Pediatr 2015; 166: 538-44 http://dx.doi.org/10.1016/j.jpeds.2014.09.041

[6] Lee JW, Davis JM. Future applications of antioxidants in premature infants. Curr Opin Pediatr 2011; 23: 161-6. http://dx.doi.org/10.1097/MOP.0b013e3283423e51

[7] Skouroliakou M, Konstantinou D, Koutri K, et al. A doubleblind, randomized clinical trial of the effect of omega-3 fatty acids on the oxidative stress of preterm neonates fed through parenteral nutrition. Eur J Clin Nutr 2010; 64: 940-7. http://dx.doi.org/10.1038/ejcn.2010.98

[8] Arboleya S, Solís G, Fernández N, de los Reyes-Gavilán CG, Gueimonde M. Facultative to strict anaerobes ratio in the preterm infant microbiota: a target for intervention? Gut Micro 2012; 3: 583-8.

http://dx.doi.org/10.4161/gmic.21942

[9] Michelland RJ, Combes S, Monteils V, Cauquil L, Gidenne T, Fortun-Lamothe L. Molecular analysis of the bacterial community in digestive tract of rabbit. Anaerobe 2010; 16: 61-5.

http://dx.doi.org/10.1016/j.anaerobe.2009.05.002

[10] Harris JK, El Kasmi KC, Anderson AL, et al. Specific microbiome changes in a mouse model of parenteral nutrition associated liver injury and intestinal inflammation. PLoS One 2014; 9: e110396. http://dx.doi.org/10.1371/journal.pone.0110396

[11] Skouroliakou $M$, Konstantinou $D$, Papasarantopoulos $P$, Matthaiou Ch. Computer assited total parenteral nutrition for preterm and sick term neonates. Pharm World Sci 2005; 27: 305-10. http://dx.doi.org/10.1007/s11096-005-2462-x

[12] Skouroliakou M, Konstantinou D, Agakidis C, et al. Cholestasis, bronchopulmonary dysplasia, and lipid profile in preterm infants receiving MCT/ $\omega$-3-PUFA-containing or soybean-based lipid emulsions. Nutr Clin Pract 2012; 27: 817-24. http://dx.doi.org/10.1177/0884533612454547

[13] Shoji H, Koletzko B. Oxidative stress and antioxidant protection in the perinatal period. Curr Opin Clin Nutr Metab Care 2007; 10: 324-8. http://dx.doi.org/10.1097/MCO.0b013e3280a94f6d

[14] Winter SE, Lopez CA, Baumler AJ.The dynamics of gutassociated microbial communities during inflammation. EMBO Reports 2013; 14: 319-27. http://dx.doi.org/10.1038/embor.2013.27

Received on 29-04-2015

Accepted on 13-05-2015

Published on 01-06-2015

\section{http://dx.doi.org/10.6000/1929-4247.2015.04.02.4}

(C) 2015 Arboleya et al.; Licensee Lifescience Global.

This is an open access article licensed under the terms of the Creative Commons Attribution Non-Commercial License (http://creativecommons.org/licenses/by-nc/3.0/) which permits unrestricted, non-commercial use, distribution and reproduction in any medium, provided the work is properly cited. 